Journal of the Persian Gulf

(Marine Science)/Vol. 8/No. 27/ March 2017/10/1-10

\title{
Investigation on the sloshing effect in a 2D tank under harmonic excitation using Smoothed Particle Hydrodynamics (SPH) and Finite Volume method (FVM)
}

\author{
Soheil Radfar ${ }^{1}$, Amir Taherkhani ${ }^{2}{ }^{2}$, Hassan Akbari ${ }^{3}$ \\ 1-PhD Candidate, Tarbiat Modares University; soheil.radfar@modares.ac.ir \\ 2-MSc Student, Tarbiat Modares University; amir.taherkhani@modares.ac.ir \\ 3-Assistant Professor, Tarbiat Modares University; akbari.h@modares.ac.ir
}

Received: November 2016

Accepted: March 2017

C) 2017 Journal of the Persian Gulf. All rights reserved.

\begin{abstract}
Sloshing describes liquids motion in the semi-filled tanks, and exerts dynamic loading on its walls. This effect is of great importance in a number of dynamic systems e.g. aerospace vehicles, road tankers, liquefied natural gas carriers, elevated water towers and petroleum cylindrical tanks. Pressures insert impacts which are important for structural strength evaluation and its correct assessment is challenging for designers because of high share of nonlinear effects, complicated changes of free surface, strong impact phenomenon and air trapping effects. Since, numerical capabilities of the Smoothed Particle Hydrodynamics (SPH) method allow proper simulation of the fluid and/or the free surface we used it to investigate the behavior of a water tank exposed to a harmonic excitation. Besides, a Finite Volume (FV) analysis was employed using FLOW3D software, for the further comparison of the results from the SPH method. To assess the results, temporal variations of the impact pressure at the specific height of the tank wall is compared against the results from the experiment and also, the Finite Difference (FD) method. The comparison showed strong correlation of SPH results with the experimental ones. But, FLOW3D is not sufficiently capable of estimating the trend of pressure time history especially near the extremes.
\end{abstract}

Keywords: Sloshing, FDM, FVM, SPH

\section{Introduction}

Sloshing is the oscillation phenomenon caused by the motion of the tank. This effect is of great importance in a number of dynamic systems e.g. aerospace vehicles, road tankers, liquefied natural

*Email: amir.taherkhani@modares.ac.ir gas carriers, elevated water towers and petroleum cylindrical tanks. For example, in the transformation of a liquid cargo, sloshing affects the stability of the carrier and may lead to structural fatigue and deterioration. Therefore, it is necessary to diminish the sloshing effect before causing the large amplitude resonance. As such, this phenomenon was the subject of many analytical, numerical and experimental 
studies. Regarding the analytical studies, Abramson (1966) utilized potential theory to investigate linear small amplitude sloshing [1]. Because of the importance of nonlinear effects, Valtinsen (1974) analyzed nonlinear sloshing using perturbation theory [2]. He also proposed a nonlinear numerical method for investigation of sloshing in 2D tanks [3]. Nakayama and Washizu (1980) used boundary element method to analyze sloshing in tanks under torsional oscillation [4]. Wu et al. (1998) calculated sloshing waves in a 3D tank using Finite Element Method (FEM) based on fully nonlinear potential theory [5]. Chen and Nokes (2005) simulated full 2D sloshing motion using Finite Difference Method (FDM) [6]. Later, Wu and Chen (2009) simulated fluid sloshing in a 3D tank with six degrees of freedom, based on this method [7]. Kim (2001) utilized SOLA scheme to solve Navier-Stokes equations and assumed that the free surface profile is one-sided conjugation [8]. Celebi and Akyildiz (2002) calculated sloshing of viscous liquids in the semi-filled rectangular tank [9]. They used FDM for the solution of equations and Volume of Fluid (VOF) for capturing the free surface. Wang and Khoo (2005) studied random nonlinear 2D excitation using FEM [10]. Sriram et al. (2006) investigated random sloshing under both horizontal and vertical excitations [11]. Theoretically, Faltinsen et al. (2000, 2001) developed next infinite modal technic, which is capable of description of the nonlinear sloshing in incompressible fluids, in which, free surface motion is expanded using Fourier series [12, 13]. Wu (2007) also analyzed the resonance of the second order sloshing [14].

Combined numerical and experimental study has also been performed in this regard. Akyildiz and Unal (2005) investigated pressure variations in both baffled- and unbaffled tank [15]. Pal et al. (1999) also studied 2D sloshing numerically and experimentally [16]. In their study, numerical results for sloshing-induced height of amplitudes and frequencies of different excitations, compared against experimental results. Of recent studies, $\mathrm{Xu}$ et al. (2017) studied the sloshing effect in membranetype LNG tanks [17]. Storey (2016) investigated the sloshing of liquid nitrogen in spherical tanks, numerically, analytically and experimentally [18].

In this paper, temporal variations of impact pressure in a specific height of a 2D tank wall subjected to harmonic rotational motion was investigated using SPH method and compared with the results of experiments, FVM and FDM. In this regard, in Section 2, basics of SPH method has been discussed. In Section 3, experimental work of Akyildiz and Unal (2005) is presented. The FDM study of Akyildiz (2012) is presented in Section 4. Finally, the results from these four categories are compared against each other.

\section{Material and Methods}

\subsection{Smoothed Particle Hydrodynamics method}

SPH method, introduced by Lucy and Monagan in 1970s, is based on Lagrangian particles method. In the SPH, for a specific flow variable in an arbitrary point with positon vector $r$ and neighboring vector $r$, Kernel approximation is as follows:

$$
\langle f(x)\rangle=\int_{D} f\left(x^{\prime}\right) W\left(x-x^{\prime}, h\right) d x
$$

Where, $W$ is Kernel function, $h$ is smoothing length or influence length of $\mathrm{W}$ function, and $D$ is the integral volume which $\mathrm{x}$ encompasses. Due to high numerical stability and being smooth, in this study, 5th order Vendland Kernel function is used. It is formulated in Eq. (2).

$W(r, h)=\alpha_{D} \cdot e^{-r^{2}}$

Where, $r=\left|x-x^{\prime}\right|$, and $q=r / h$.In the equation, 
$\alpha_{D}$ is $7 / 4 \pi h^{2}$ for $2 \mathrm{D}$ models.

In order to solve the problem, the computational domain has to be descretized on several particles (see Figure 1). Each one of the particles represents a limited volume of fluid. Therefore, Eq. (1) can be rewritten as,

$$
\left\langle f\left(x_{i}\right)\right\rangle=\sum_{j=1}^{N} f\left(x_{j}\right) W\left(x_{i}-x_{j}, h_{i j}\right) \Delta V_{j}
$$

Here, $\Delta V_{j}=m_{j} / \rho_{j}$ is the corresponding volume of fluid of the particle $j$.

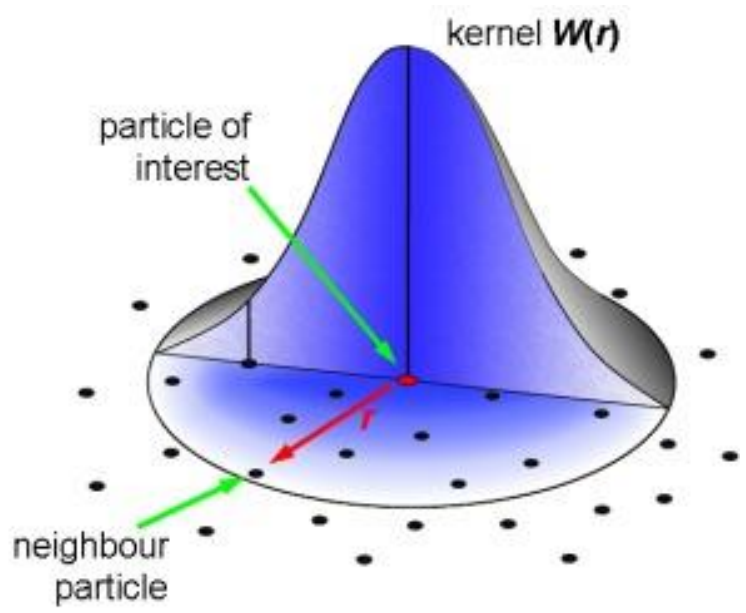

Fig 1: Kernel approximation in a smoothed neighbor

Using this formulation, gradient of a field function can be presented as,

$$
\left\langle\nabla f\left(x_{i}\right)\right\rangle=\frac{1}{\rho_{i}} \sum_{j=1}^{N} m_{j}\left[f\left(x_{j}\right)-f\left(x_{i}\right)\right] \nabla_{i} W_{i j}
$$

where, the gradient of Kernel function is defined as,

$$
\nabla_{i} W_{i j}=\frac{x_{i}-x_{j}}{r_{i j}} \frac{\partial W_{i j}}{\partial r_{i j}}=\frac{x_{i j}}{r_{i j}} \frac{\partial W_{i j}}{\partial r_{i j}}
$$

Internal forces are calculated based on mass and momentum conservation equations (i.e. Navier-
Stokes equations):

$$
\begin{aligned}
& \frac{D \rho}{D t}=-\rho \nabla \cdot v \\
& \frac{D v}{D t}=g+\frac{1}{\rho} \nabla \cdot \sigma
\end{aligned}
$$

In the mass conservation equation (Eq. (6)), fluid is assumed to be weekly compressible in the entire domain. In the continuity equation, $\sigma$ is stress tensor and is defined as follows:

$$
\sigma=-p \delta+\tau=-p \delta+\mu \varepsilon
$$

Where, stress rate tensor $\varepsilon$ is formulated as,

$$
\varepsilon^{l m}=\left(\frac{\partial v^{m}}{\partial x^{l}}+\frac{\partial v^{l}}{\partial x^{m}}\right)-\frac{2}{3}(\nabla \cdot v) \cdot \delta^{l m}
$$

Here, $\delta$ is Kronecker Delta function and is,

$\delta^{l m}= \begin{cases}1 & l=m \\ 0 & l \neq m\end{cases}$

There are various forms of mass conservation equation in discrete approximation of smoothed particles. In the current paper, the $i$ particle is the reference and the $j$ particle represents the particles within the domain. Hence, discrete form of mass conservation equation is exerted as follows,

$$
\frac{D \rho_{i}}{D t}=-\sum_{j=1}^{N} m_{j} v_{i j} . \nabla W_{i j}, \quad v_{i j}=\left(v_{i}-v_{j}\right)
$$

Navier-Stokes equation can be approximated as,

$\frac{D v_{i}}{D t}=-\sum_{j=1}^{N} m_{j}\left(\frac{\sigma_{i}+\sigma_{j}}{\rho_{i} \rho_{j}}\right) \cdot \nabla W_{i j}$

Where, general stress-strain tensor is calculated as follows: 


$$
\begin{aligned}
\sigma_{i}= & p_{i} \delta+\mu_{i} \varepsilon_{i} \\
\varepsilon_{i}^{l m}= & \sum_{j=1}^{N} \frac{m_{j}}{\rho_{j}} v_{i j}^{m} \frac{\partial W_{i j}}{\partial x_{i}^{l}}+\sum_{j=1}^{N} \frac{m_{j}}{\rho_{j}} v_{i j}^{l} \frac{\partial W_{i j}}{\partial x_{i}^{m}} \\
& -\frac{2}{3}\left(\sum_{j=1}^{N} \frac{m_{j}}{\rho_{j}} v_{i j} \cdot \nabla_{i} W_{i j}\right) \delta^{l m}
\end{aligned}
$$

In this study, effect of viscous term is neglected; hence, Euler equations may be used. These equations have been successfully employed for sloshing simulation by Souto-Iglesias et al. (2006) [22] .

Besides, equation of state for weekly compressible fluid is defined as Eq. (14) and this equation is solved to find pressure of each particle, which density was previously calculated in Eq. (11).

$$
p=\frac{c_{0}^{2} \rho_{0}}{\gamma}\left[\left(\frac{\rho}{\rho_{0}}\right)^{\gamma}-1\right]
$$

Where, power coefficient $\gamma$ is adopted 7 according to Batchelor (2000) proposal [23].

\subsection{Experimental Study}

In this paper, experimental work of Akyildiz and Unal (2005) is used for controlling the numerical result. They experimentally investigated the 3D effects of liquid sloshing loading. Since, the rotation was around the perpendicular axis, the problem can be investigated in 2D. Figure 2 shows the tank geometry and the locations of transducers.

Here, the authors just compared the results of wall pressure in the location T1, when tank is filled to half of its total height. Tank motion is rotationally harmonic around the perpendicular axis. The harmonic motion obeys the sinusoidal form $\theta_{R}=\theta_{0} \sin (\omega . t)$, where $\theta_{0}$ and $\omega$ are amplitude and frequency of rotation, respectively. In the present study, these values are 8 degrees and $2 \mathrm{rad} / \mathrm{s}$, respectively. Figure 3 shows the time history of the roll amplitude $\left(\theta_{0}\right)$.

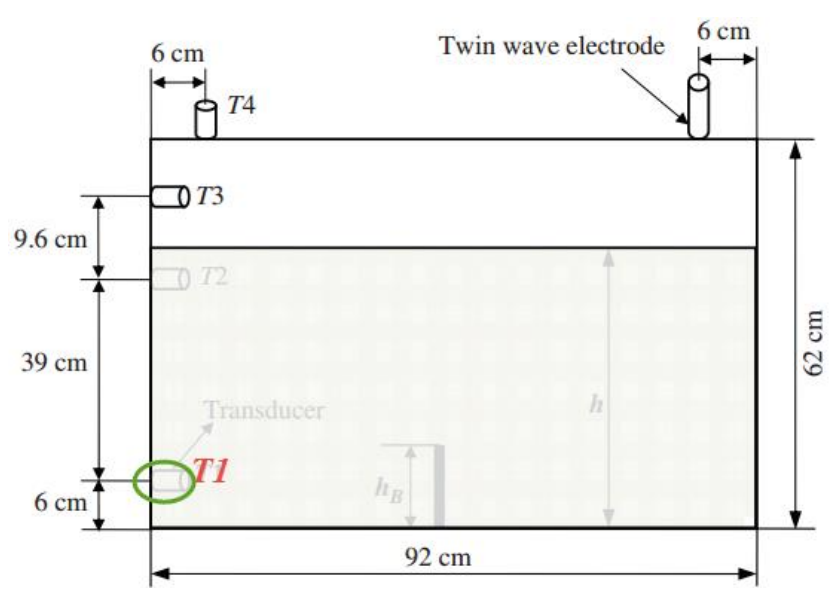

Fig 2: Geometry of the tank and the location of transducer $\mathbf{T 1}$

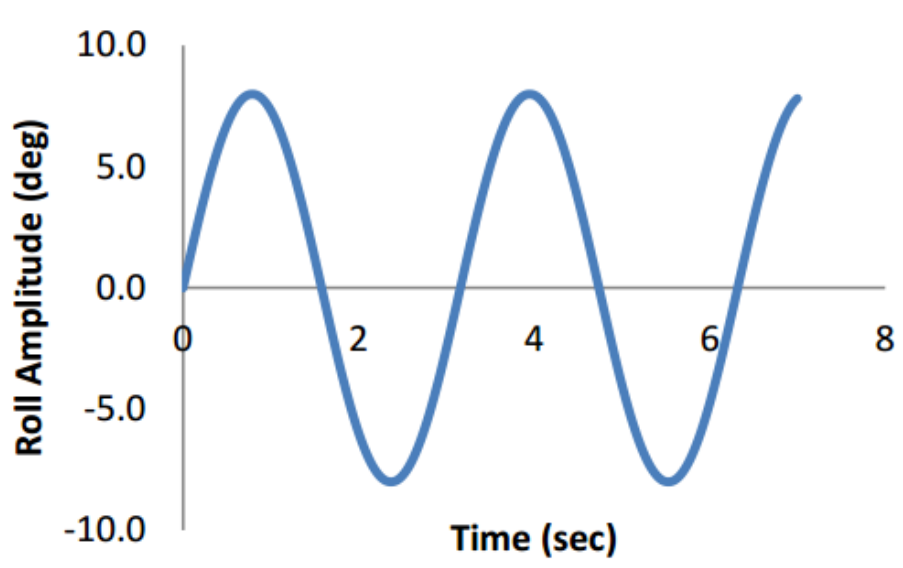

Fig 3: Time history of the roll amplitude

\subsection{Finite Difference Study}

Akyildiz (2012) used a numerical scheme based on volume of fluid technic for investigation of nonlinear liquid sloshing behavior [19]. In his study, full Navier-Stokes equations are solved using finite difference technics in the moving coordinate system. Effects of turbulence and wave breaking are neglected in his study. 


\subsection{Finite Volume Study}

For further investigations, a FV analysis has been performed using FLOW3D commercial software. FLOW3D uses FVM for the numerical solution of fluid governing equation and simulates free surface of the fluid using Volume of Fluid (VOF) method. Momentum and continuity equations are the governing equations, here. Eq. (15) shows the continuity equation:

$$
\frac{\partial \rho}{\partial t}+\nabla \cdot(\rho \vec{U})=0
$$

Where $\rho$ is fluid density and $\vec{U}$ is the vector of fluid velocity. Since, the fluid is assumed to be incompressible, fluid density is constant during the runtime. Therefore, the above equation can be rewritten as,

$$
\nabla(\vec{U})=0
$$

Besides, the governing equations of fluid motion for velocity components along $\mathrm{x}, \mathrm{y}$ and $\mathrm{z}$ are NavierStokes equations:

$$
\frac{\partial \vec{U}}{\partial t}+\vec{U} \cdot(\nabla \vec{U})-v \nabla^{2} \vec{U}+\frac{1}{\rho} \nabla P+\vec{F}=0
$$

Where, $v$ is the kinematic viscosity of fluid, $\rho$ is the fluid density, $P$ is the fluid pressure and $\vec{F}$ is the vector of external forces acting on the fluid.

In the modelling of sloshing phenomenon using FLOW3D by performing mesh independency analysis, the CFD domain is finally divided into 10,000 uniform computational cells.

\section{Results and Discussion}

To obtain appropriate results in SPH modelling; the parameters are valued based on Table 1. In this simulation, distance between the particles is adopted $0.002 \mathrm{~m}$. Total number of fluid and boundary particles are 70686 and 1542, respectively. The run time of the simulation is approximately 71 minutes. The model is implemented within the open source code DualSPHysics4 v4.0.055 and the system had the graphic card of NVIDA GTX $750 \mathrm{Ti}$ with five cores and $1.1 \mathrm{GHz}$.

Table 1: Numerical settings for SPH method

\begin{tabular}{ll}
\hline Temporal integration scheme & Verlet algorithm \\
Kernel function & Wendland \\
Viscous behavior & Artificial viscosity \\
Equation of state & Tait equation \\
\hline
\end{tabular}

Figure 4 shows contours of velocity magnitude in four time steps. With leftward motion of the tank from the stable position; the liquid within the tank compresses the left wall and thus, the pressure of the wall increases. Reaching the maximum roll motion (here, 8 degrees), tank velocity becomes zero and it starts rolling reverse. At the time, the liquid has still leftward motion. This causes the sloshing wave to hit the opposite wall. Therefore, the sloshing wave starts rightward motion. After reaching the wave to the right wall and hitting to it, the leftward motion of the tank is started. Generally, in the beginning of rolling motion, there is a specific phase difference between tank motion and wave sloshing. However, due to forced rolling motion of the tank, gradually, motion of the sloshing wave and the tank is being coordinated. 

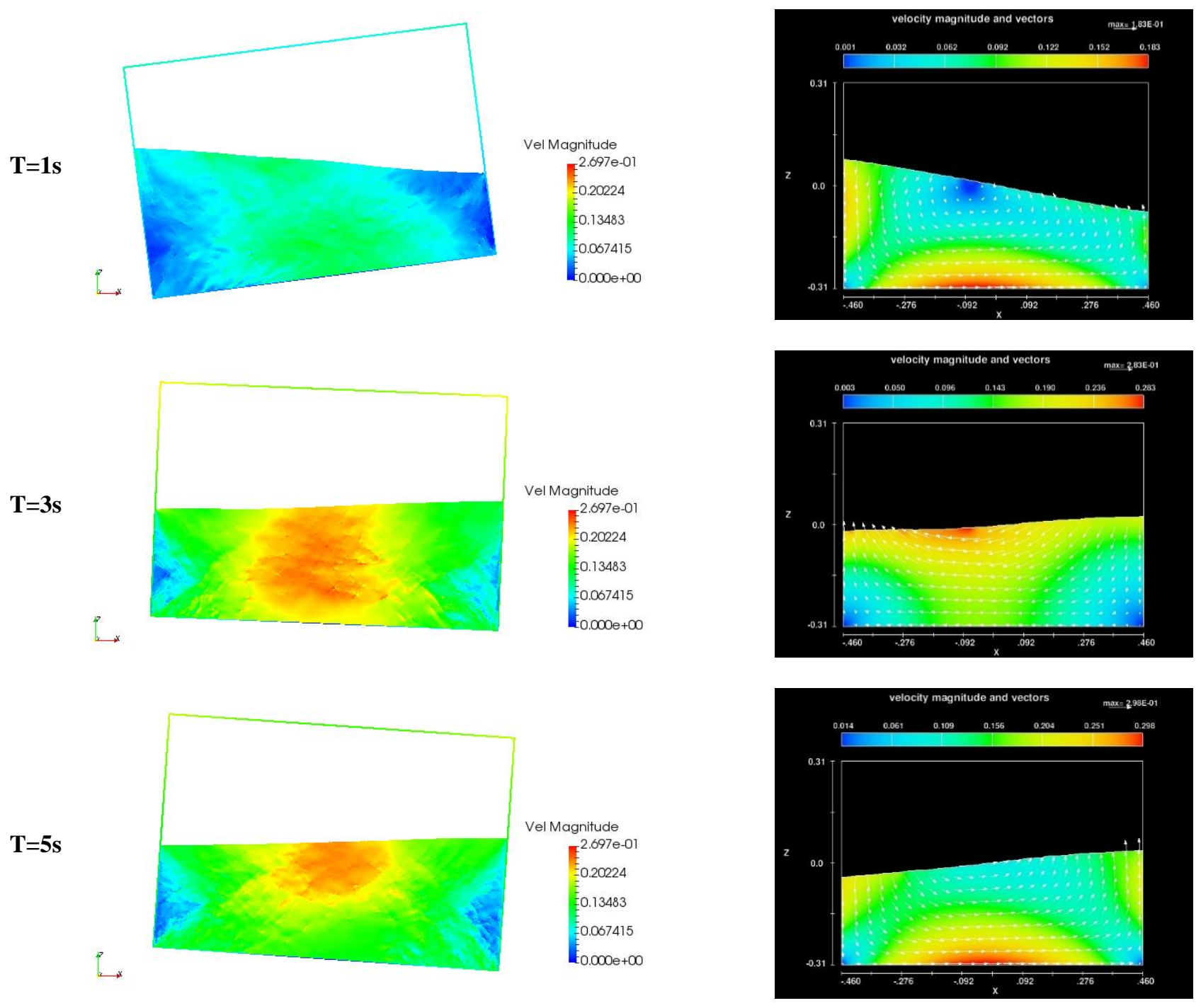

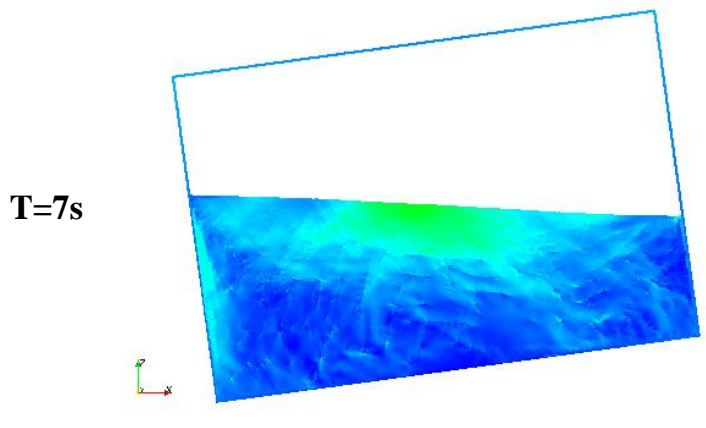

(a) SPH

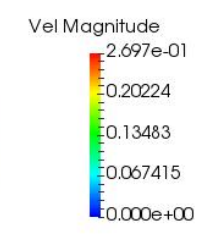

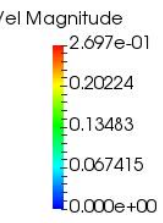

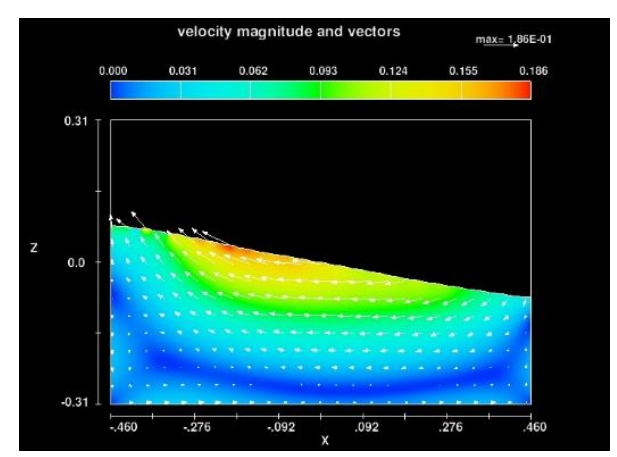

(b) FVM

Fig 4: Contours of velocity magnitudes for two methods;

(a) SPH and (b) FVM 
Figure 5 shows the comparison between the results of four above mentioned methods, for pressure variations of the left wall at the height $6 \mathrm{~cm}$ from the tank floor (T1 position in Figure 2). As can be seen, local maximums of pressure time history in the SPH method, is underestimated comparing with experiments; but, this method has significantly good correlation with benchmark results in the local minimums. For the simulation with FLOW3D, although, the results are acceptable, it is not sufficiently capable of estimating the trend of time history, especially near the extremes.

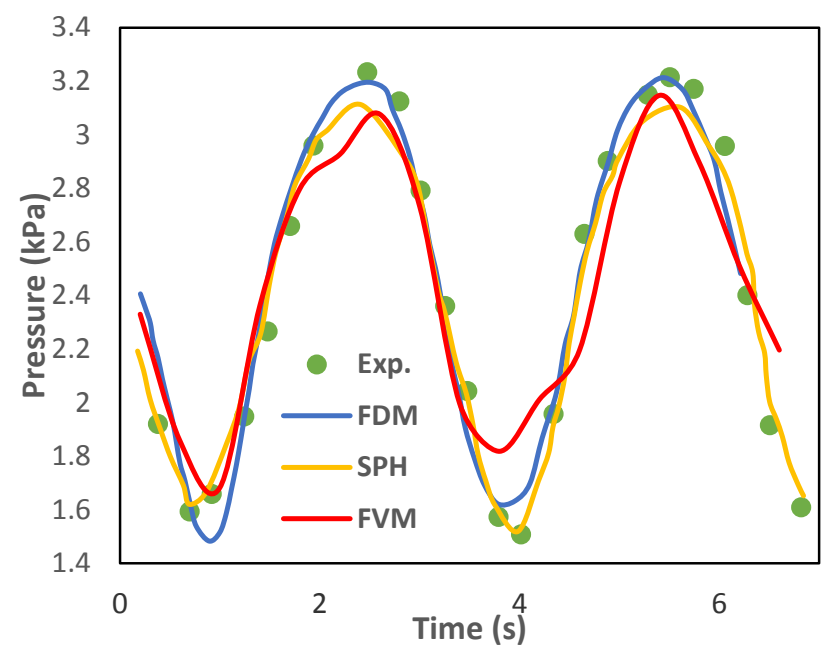

Fig 5: Pressure variations at location T1 of the left wall

\section{Conclusions}

In the present study, pressure variations in a specific height of the wall of a 2D tank were investigated. The tank is excited to harmonic roll motion. Numerical simulations were carried out using SPH and FVM methods and the results compared against experimental (Akyildiz and Unal [15]) and FDM (Akyildiz [19]) results. Comparisons on the pressure variation time history showed that SPH has more accuracy to match the local minimums than FDM and FVM. However, it underestimates the local maximums compared with
FDM results. Besides, results of the FVM study clearly indicates that FLOW3D is not sufficiently capable of estimating the trend of pressure time history, especially near the extremes. Therefore, further study is necessary to calibrate the settings of the software to obtain more reasonable correlation with experimental results.

\section{References}

Abramson, H. N., The dynamic behavior of liquids in moving containers, with applications to space vehicle technology, 1966.

Valtinsen O., "A nonlinear theory of sloshing in rectangular tanks," Journal of Ship Research, vol. 18, 1974.

Faltinsen, O. M., "A numerical nonlinear method of sloshing in tanks with two-dimensional flow," Journal of Ship Research, vol. 22, 1978.

Nakayama, T., and Washizu, K., "Nonlinear analysis of liquid motion in a container subjected to forced pitching oscillation," International Journal for Numerical Methods in Engineering, vol. 15, pp. 1207-1220, 1980. https://doi.org/10.1002/nme.1620150808

Wu, G., Ma, Q., and Taylor, R. E., "Numerical simulation of sloshing waves in a 3D tank based on a finite element method," Applied Ocean Research, vol. 20, pp.37-355, 1998. https://doi.org/10.1016/S0141-1187(98)00030-3

Chen, B. F., and Nokes, R., "Time-independent finite difference analysis of fully non-linear and viscous fluid sloshing in a rectangular tank," Journal of Computational Physics, vol. 209, pp. 47-81, 2005. https://doi.org/10.1016/j.jcp.2005.03.006

C.-H. $\mathrm{Wu}$ and B.-F. Chen, "Sloshing waves and resonance modes of fluid in a $3 \mathrm{D}$ tank by a timeindependent finite difference method," Ocean Engineering, vol. 36, pp. 500-510, 2009. https://doi.org/10.1016/j.oceaneng.2009.01.020 
Y. Kim, "Numerical simulation of sloshing flows with impact load," Applied Ocean Research, vol. 23, pp. 53-62, 2001. https://doi.org/10.1016/S0141-1187(00)00021-3

M. S. Celebi and H. Akyildiz, "Nonlinear modeling of liquid sloshing in a moving rectangular tank," Ocean Engineering, vol. 29, pp. 1527-1553, 2002. https://doi.org/10.1016/S0029-8018(01)00085-3

C. Wang and B. Khoo, "Finite element analysis of two-dimensional nonlinear sloshing problems in random excitations," Ocean Engineering, vol. 32, pp. 107-133, 2005.

https://doi.org/10.1016/j.oceaneng.2004.08.001

V. Sriram, S. Sannasiraj, and V. Sundar, "Numerical simulation of 2D sloshing waves due to horizontal and vertical random excitation," Applied Ocean Research, vol. 28, pp. 19-32, 2006. https://doi.org/10.1016/j.apor.2006.01.002

O. M. Faltinsen, O. F. Rognebakke, I. A. Lukovsky, and A. N. Timokha, "Multidimensional modal analysis of nonlinear sloshing in a rectangular tank with finite water depth," Journal of Fluid Mechanics, vol. 407, pp. 201-234, 2000. https://doi.org/10.1017/S0022112099007569

O. M. Faltinsen and A. N. Timokha, "An adaptive multimodal approach to nonlinear sloshing in a rectangular tank," Journal of Fluid Mechanics, vol. 432, pp. 167-200, 2001. https://doi.org/10.1017/S0022112000003311

G. Wu, "Second-order resonance of sloshing in a tank," Ocean engineering, vol. 34, pp. 2345- 2349, 2007.

https://doi.org/10.1016/j.oceaneng.2007.05.004

H. Akyildiz and E. Ünal, "Experimental investigation of pressure distribution on a rectangular tank due to the liquid sloshing," Ocean Engineering, vol. 32, pp. 1503-1516, 2005.

https://doi.org/10.1016/j.oceaneng.2004.11.006
N. Pal, S. Bhattacharyya, and P. Sinha, "Coupled slosh dynamics of liquid-filled, composite cylindrical tanks," Journal of engineering mechanics, vol. 125, pp. 91-495, 1999. https://doi.org/10.1061/(ASCE)07339399(1999)125:4(491)

J. Xu, J. Wang, and M. Souli, "SPH and ALE formulations for sloshing tank analysis," The International Journal of Multiphysics, vol. 9, 2016. https://doi.org/10.1260/1750-9548.9.3.209

J. M. Storey, "Experimental, Numerical, and Analytical Slosh Dynamics of Water and Liquid Nitrogen in a Spherical Tank," Florida Institute of Technology, 2016.

H. Akyildiz, "A numerical study of the effects of the vertical baffle on liquid sloshing in two dimensional rectangular tank," Journal of Sound and Vibration, vol. 331, pp. 41-52, 2012. https://doi.org/10.1016/j.jsv.2011.08.002

J. J. Monaghan, "Smoothed particle hydrodynamics," Annual review of astronomy and astrophysics, vol. 30, pp. 543-574, 1992. https://doi.org/10.1146/annurev.aa.30.090192.002 $\underline{551}$

M. Gomez-Gesteira, B. D. Rogers, A. J. Crespo, R. A. Dalrymple, M. Narayanaswamy, and J. M. Dominguez, "SPHysics-development of a free surface fluid solver-Part 1: Theory and formulations," Computers \& Geosciences, vol. 48, pp. 289-299, 2012.

https://doi.org/10.1016/j.cageo.2012.02.029

A. Souto-Iglesias, L. Delorme, L. Pérez-Rojas, and S. Abril-Pérez, "Liquid moment amplitude assessment in sloshing type problems with smooth particle hydrodynamics," Ocean Engineering, vol. 33, pp. 1462-1484, 2006. https://doi.org/10.1016/j.oceaneng.2005.10.011

Abramson, H. N., The dynamic behavior of liquids 
in moving containers, with applications to space vehicle technology, 1966.

Valtinsen O., "A nonlinear theory of sloshing in rectangular tanks," Journal of Ship Research, vol. 18, 1974.

Faltinsen, O. M., "A numerical nonlinear method of sloshing in tanks with two-dimensional flow," Journal of Ship Research, vol. 22, 1978.

Nakayama, T., and Washizu, K., "Nonlinear analysis of liquid motion in a container subjected to forced pitching oscillation," International Journal for Numerical Methods in Engineering, vol. 15, pp. 1207-1220, 1980.

\section{https://doi.org/10.1002/nme.1620150808}

Wu, G., Ma, Q., and Taylor, R. E., "Numerical simulation of sloshing waves in a 3D tank based on a finite element method," Applied Ocean Research, vol. 20, pp.37-355, 1998. https://doi.org/10.1016/S0141-1187(98)00030-3

Chen, B. F., and Nokes, R., "Time-independent finite difference analysis of fully non-linear and viscous fluid sloshing in a rectangular tank," Journal of Computational Physics, vol. 209, pp. 47-81, 2005. https://doi.org/10.1016/j.jcp.2005.03.006

C.-H. Wu and B.-F. Chen, "Sloshing waves and resonance modes of fluid in a 3D tank by a timeindependent finite difference method," Ocean Engineering, vol. 36, pp. 500-510, 2009. https://doi.org/10.1016/j.oceaneng.2009.01.020

Y. Kim, "Numerical simulation of sloshing flows with impact load," Applied Ocean Research, vol. 23, pp. 53-62, 2001. https://doi.org/10.1016/S0141-1187(00)00021-3

M. S. Celebi and H. Akyildiz, "Nonlinear modeling of liquid sloshing in a moving rectangular tank," Ocean Engineering, vol. 29, pp. 1527-1553, 2002. https://doi.org/10.1016/S0029-8018(01)00085-3
C. Wang and B. Khoo, "Finite element analysis of two-dimensional nonlinear sloshing problems in random excitations," Ocean Engineering, vol. 32, pp. 107-133, 2005.

https://doi.org/10.1016/j.oceaneng.2004.08.001

V. Sriram, S. Sannasiraj, and V. Sundar, "Numerical simulation of 2D sloshing waves due to horizontal and vertical random excitation," Applied Ocean Research, vol. 28, pp. 19-32, 2006. https://doi.org/10.1016/j.apor.2006.01.002

O. M. Faltinsen, O. F. Rognebakke, I. A. Lukovsky, and A. N. Timokha, "Multidimensional modal analysis of nonlinear sloshing in a rectangular tank with finite water depth," Journal of Fluid Mechanics, vol. 407, pp. 201-234, 2000. https://doi.org/10.1017/S0022112099007569

O. M. Faltinsen and A. N. Timokha, "An adaptive multimodal approach to nonlinear sloshing in a rectangular tank," Journal of Fluid Mechanics, vol. 432, pp. 167-200, 2001. https://doi.org/10.1017/S0022112000003311

G. Wu, "Second-order resonance of sloshing in a tank," Ocean engineering, vol. 34, pp. 2345- 2349, 2007.

https://doi.org/10.1016/j.oceaneng.2007.05.004

H. Akyildiz and E. Ünal, "Experimental investigation of pressure distribution on a rectangular tank due to the liquid sloshing," Ocean Engineering, vol. 32, pp. 1503-1516, 2005. https://doi.org/10.1016/j.oceaneng.2004.11.006

N. Pal, S. Bhattacharyya, and P. Sinha, "Coupled slosh dynamics of liquid-filled, composite cylindrical tanks," Journal of engineering mechanics, vol. 125, pp. 91-495, 1999. https://doi.org/10.1061/(ASCE)07339399(1999)125:4(491)

J. Xu, J. Wang, and M. Souli, "SPH and ALE formulations for sloshing tank analysis," The 
International Journal of Multiphysics, vol. 9, 2016. https://doi.org/10.1260/1750-9548.9.3.209

J. M. Storey, "Experimental, Numerical, and Analytical Slosh Dynamics of Water and Liquid Nitrogen in a Spherical Tank," Florida Institute of Technology, 2016.

H. Akyildiz, "A numerical study of the effects of the vertical baffle on liquid sloshing in two dimensional rectangular tank," Journal of Sound and Vibration, vol. 331, pp. 41-52, 2012.

https://doi.org/10.1016/j.jsv.2011.08.002

J. J. Monaghan, "Smoothed particle hydrodynamics," Annual review of astronomy and astrophysics, vol. 30, pp. 543-574, 1992.

https://doi.org/10.1146/annurev.aa.30.090192.002 $\underline{551}$
M. Gomez-Gesteira, B. D. Rogers, A. J. Crespo, R. A. Dalrymple, M. Narayanaswamy, and J. M. Dominguez, "SPHysics-development of a free surface fluid solver-Part 1: Theory and formulations," Computers \& Geosciences, vol. 48, pp. 289-299, 2012.

https://doi.org/10.1016/j.cageo.2012.02.029

A. Souto-Iglesias, L. Delorme, L. Pérez-Rojas, and S. Abril-Pérez, "Liquid moment amplitude assessment in sloshing type problems with smooth particle hydrodynamics," Ocean Engineering, vol. 33, pp. 1462-1484, 2006.

https://doi.org/10.1016/j.oceaneng.2005.10.011

G. K. Batchelor, An introduction to fluid dynamics: Cambridge university press, 2000. https://doi.org/10.1017/CBO9780511800955 